\title{
A assistência de enfermagem frente ao paciente oncológico: uma revisão integrativa
}

\author{
Nursing care for cancer patients: an integrative review \\ Atención de enfermería para pacientes con cáncer: una revisión integradora
}

Anna Carolina Lomelino Lemos Medeiros ORCID: https://orcid.org/0000-0002-8835-9389

Universidade de Vassouras, Brasil e-mail: carolinalomelino@outlook.com

Aida Francisca da Silva

ORCID: https://orcid.org/0000-0002-2990-8480

Universidade Iguaçu, Brasil

e-mail: aydaenfermagem2016@gmail.com

Bruna Abas dos Santos

ORCID: https://orcid.org/0000-0001-7304-9823 Universidade Estácio de Sá, Brasil e-mail: bruna.abas@edu.unirio.br

Dayana Campos de Oliveira Cardoso ORCID: https://orcid.org/0000-0002-5031-6041 Universidade Estácio de Sá, Brasil

e-mail: camposdayana2015@hotmail.com

Débora Maria da Silva Xavier

ORCID: https://orcid.org/0000-0002-1381-544X

Universidade Federal de Pernambuco, Brasil e-mail: debxavier@hotmail.com

Vivian Larissa Batista Lemos

ORCID: https://orcid.org/0000-0003-1782-9168 Centro Universitário Estácio de Sergipe, Brasil e-mail: lemosvivian1@gmail.com

Gabriela Alves Santana de Oliveira ORCID: https://orcid.org/0000-0002-9530-0924 Universidade Iguaçu, Brasil

e-mail: gabrielaalvessoliveira@gmail.com

Isabella Magalhães Barbosa

ORCID: https://orcid.org/0000-0002-7499-0890

Universidade Veiga de Almeida, Brasil e-mail: issxmagalhaes@gmail.com

Larissa de Lima Nascimento Silva ORCID: https://orcid.org/0000-0002-7760-4174

Centro Universitário Braz Cubas, Brasil e-mail: larissalimansilva@gmail.com

Larissa Machado Campana Portela

ORCID: https://orcid.org/0000-0003-3248-2924

Universidade Veiga de Almeida, Brasil e-mail: larissaenfuva@hotmail.com

Lívia Luiza Gomes Barreto

ORCID: https://orcid.org/0000-0002-5289-3192

Universidade Veiga de Almeida, Brasil e-mail: livialuiza.gomes@gmail.com

Raísa Helena do Nascimento Rodrigues ORCID: https://orcid.org/0000-0001-6351-6520 Universidade de Pernambuco, Brasil e-mail: raisa.rodrigues@upe.br

Ronilce Rozendo Amorim

ORCID: https://orcid.org/0000-0003-2291-6055 Universidade Estácio de Sá, Brasil e-mail: ronilceamorym@gmail.com

Thamiris Quinzi Andrade

ORCID: https://orcid.org/0000-0001-6989-5023

Centro Universitário de Volta Redonda, Brasil e-mail: thamiris.quinzi@hotmail.com

Valéria Cristina Brandão Marinho ORCID: https://orcid.org/0000-0002-9344-6161

Faculdade de Enfermagem UNESA, Brasil e-mail: vcbmarinho@gmail.com 


\title{
Resumo
}

Introdução: Chamamos de câncer uma cadeia de doenças que têm em conjunto um desequilíbrio na evolução e divisão das células. As células anormais, podem se ampliar e adentrar outras estruturas corporais, em um decurso denominado por metástase. Objetivo: Descrever a importância da assistência de enfermagem frente a pacientes oncológicos. Metodologia: Este estudo trata-se de uma revisão integrativa de literatura, de abordagem qualitativa. Foram definidos os seguintes critérios de inclusão: artigos indexados na íntegra e disponíveis para leitura, em Língua Portuguesa e texto completo, com recorte temporal de 5 anos (2016 a 2021). Como critérios de exclusão foram descartados os artigos em repetição ou que não estavam em concordância com a temática proposta. Resultados e discussões: A enfermagem tem em como fundamento o dever com a integralidade, o qual se realiza através do amparo, conexão e comunicação e se segura no reconhecimento das insuficiências na assistência exposta pelos usuários e sua família, por ser uma ação centralizada no indivíduo e não exclusivamente na metodologia. Considerações finais: A área oncológica é uma especialização constituída por diversos obstáculos desde a investigação, cirurgias, modelos terapêuticos e as contínuas inovações médicas, o que exige uma preparação dos profissionais de saúde, pois é de grande complexidade científica e técnica.

Palavras-chave: Enfermagem; Oncologia; Assistência.

\begin{abstract}
Introduction: We call cancer a chain of diseases that have together an imbalance in cell evolution and division. Abnormal cells can expand and enter other body structures, in a course called metastasis. Objective: To describe the importance of nursing care for cancer patients. Methodology: This study is an integrative literature review with a qualitative approach. The following inclusion criteria were defined: articles indexed in full and available for reading, in Portuguese and full text, with a time frame of 5 years (2016 to 2021). As exclusion criteria, repeated articles or articles that were not in accordance with the proposed theme were discarded. Results and discussions: Nursing is based on the duty to comprehensiveness, which is carried out through support, connection and communication and is assured in the recognition of insufficiencies in care exposed by users and their families, as it is an action centered on the individual and not exclusively in the methodology. Final considerations: The oncology area is a specialization constituted by several obstacles from research, surgeries, therapeutic models and continuous medical innovations, which requires the preparation of health professionals, as it is of great scientific and technical complexity.
\end{abstract}

Keywords: Nursing; Oncology; Assistance.

\section{Resumen}

Introducción: Llamamos cáncer a una cadena de enfermedades que tienen juntas un desequilibrio en la evolución y división celular. Las células anormales pueden expandirse y entrar en otras estructuras corporales, en un curso llamado metástasis. Objetivo: Describir la importancia de la atención de enfermería al paciente oncológico. Metodología: Este estudio es una revisión integradora de la literatura con un enfoque cualitativo. Se definieron los siguientes criterios de inclusión: artículos indexados íntegramente y disponibles para lectura, en portugués y texto completo, con un plazo de 5 años (2016 a 2021). Como criterio de exclusión se descartaron los artículos repetidos o los artículos que no concordaban con la temática propuesta. Resultados y discusiones: La enfermería se fundamenta en el deber de integralidad, que se realiza a través del acompañamiento, la conexión y la comunicación y se asegura en el reconocimiento de las insuficiencias en el cuidado expuestas por los usuarios y sus familias, por ser una acción centrada en el individuo y no exclusivamente en la metodología. Consideraciones finales: El área de oncología es una especialización constituida por varios obstáculos de investigación, cirugías, modelos terapéuticos e innovaciones 
médicas continuas, que requiere la preparación de profesionales de la salud, por ser de gran complejidad científica y técnica.

Palabras clave: Enfermería; Oncología; Asistencia.

\section{Introdução}

Chamamos de câncer uma cadeia de doenças que têm em conjunto um desequilíbrio na evolução e divisão das células. As células anormais, podem se ampliar e adentrar outras estruturas corporais, em um decurso denominado por metástase. O câncer é uma adversidade de saúde comunitária, ficando em segunda posição em mortalidade mundialmente. No Brasil, com a modificação da representação de morbimortalidade dos últimos anos e mudanças sociais e culturais, ocorreu uma elevação na quantidade de mortes relacionadas às patologias crônicas. (Rolim et al., 2020).

Há carecimento do papel da enfermagem nas ações educativas, nas funções de rastreio da patologia, nas buscas e estudos científicos, nas ideologias de saúde e na formação de métodos para prevenir e detectar precocemente. Diminuindo, desta maneira, o aparecimento de novos fatos e de óbitos por câncer e contendo o que chamamos atualmente como um problema público de saúde. (Rolim et al. 2020).

Para a população, câncer é sinônimo de sofrimento. Ao receber um diagnóstico negativo perante avaliação de um nódulo, o paciente apresenta diversos comportamentos e sentimentos, como medo, angústia, agitação, negação e sofrimento. Nesse momento, a equipe de enfermagem precisa compreender as atitudes negativas do cliente e apoiá-lo nessa fase delicada da vida. (Stumm et al., 2008).

Além do desempenho de cuidados e habilidades necessárias frente ao paciente oncológico, a comunicação pode ser o ponto principal da relação entre cliente e equipe, pois é a forma mais eficaz de interação. Cabe lembrar que não precisa ser estabelecida apenas por diálogo, pode ser realizada através de gestos, cuidados; expressões faciais. Através dessa prática é possível construir vínculo com paciente, o que configura um elemento de ouro durante a assistência. (Silva \& Zago, 2005).

A pesquisa possui o objetivo de descrever a importância da assistência de enfermagem frente a pacientes oncológicos.

\section{Metodologia}

Este estudo trata-se de uma revisão integrativa de literatura, de abordagem qualitativa, o que proporciona uma síntese do conhecimento e a incorporação da aplicabilidade de resultados de estudos significativos na prática (Souza, Silva, \& Carvalho, 2010)

A pesquisa de abordagem qualitativa é um método de estudo que busca descrever e explicar os fenômenos pesquisados, através de estudos observacionais e entrevistas contextuais. Devendo esta metodologia ser estruturada a modo de responder à questão da pesquisa formulada, com uma análise lógica dos dados levantados, para fomentar uma conclusão coerente, sendo seu objetivo principal a observância dos fenômenos apresentados. (Cyriaco, Nunn, Amorim, Falcão, \& Moreno, 2017)

Bardin (2016) define que a pesquisa qualitativa é a que se fundamenta principalmente em análises qualitativas, caracterizando-se, em princípio, pela não utilização de instrumental estatístico na análise dos dados.

No entanto, para Gerhardt \& Silveira (2009), “a pesquisa descritiva tende a exigir do investigador uma gama de informações sobre o tema a ser investigado". Salienta que este tipo de estudo tem por pretensão descrever os fatos e fenômenos de determinada situação e realidade.

A proposta de construção de Revisão Integrativa de acordo com Mendes, Silveira e Galvão (2008) é um método com etapas distintas a serem seguidas. De acordo com esses critérios, esta revisão deve conter seis etapas, sendo elas: 
Estabelecimento da hipótese ou questão de pesquisa; Amostragem ou busca na literatura; Categorização dos estudos; avaliação dos estudos incluídos na revisão; Interpretação dos resultados; Síntese do conhecimento ou apresentação da revisão.

A pesquisa de abordagem qualitativa não se preocupa com o aprofundamento da compreensão de um grupo social, de uma organização, se opondo ao pressuposto que defende um modelo único de pesquisa para todas as ciências, já que as ciências sociais têm sua especificidade pressupondo uma metodologia própria. Goldenberg (1997).

Tratando-se de pesquisa descritiva, será aborda a descrição do problema investigado, o que possibilita conhecer os fenômenos investigados, aprofundando seus limites em uma realidade específica. (Polit \& Beck, 2011).

Para a construção e elaboração da questão norteadora utilizou o método voltado para a pesquisa clínica. A estratégia PICo possui um acrônimo para paciente, intervenção e contexto. Sendo elementos fundamentais na formulação da questão norteadora para assim ser realizada a busca bibliográfica. (Santos, Pimenta, \& Nobre, 2007).

Quadro 1: Estratégia PICo, para definição da questão norteadora.

\begin{tabular}{|l|l|l|}
\hline Acrônimo & Descrição & Componentes da questão \\
\hline P & Participantes & Pacientes oncológicos \\
\hline I & Intervenção & Assistência \\
\hline Co & Contexto & Assistência de enfermagem com os pacientes oncológicos \\
\hline
\end{tabular}

Fonte: Elaborada pelos autores.

O processo de interpretação dos dados é considerado a fase mais complexa, sendo necessário a iniciação do estudo com um entendimento e incorporação de definições filosóficas e epidemiológicas que estruturam a pesquisa, desde o processo de seleção do objeto de estudo da pesquisa. Minayo (2011)

Os documentos foram obtidos e escolhidos estudos por meio de coleta de dados de publicações de autores de referência na área com posterior leitura crítica dos títulos e resumos, na Biblioteca Virtual em Saúde (BVS), utilizando os seguintes Descritores em Ciência da Saúde (DESC) para a busca e seleção de dados: "Enfermagem", "Oncologia" e "Assistência", utilizando o operador booleano "AND" entre eles na pesquisa.

Foram definidos os seguintes critérios de inclusão: artigos indexados na íntegra e disponíveis para leitura, em Língua Portuguesa e texto completo, com recorte temporal de 5 anos (2016 a 2021). Como critérios de exclusão foram descartados os artigos em repetição ou que não estavam em concordância com a temática proposta. Após empregar os critérios de inclusão e exclusão na busca bibliográfica, foi possível observar uma imensa escassez de publicações e estudos referentes a temática, emergindo um total de 09 artigos para compor este trabalho, alinhados ao tema proposto.

\section{Resultados e Discussão}

Os resultados apresentados no estudo foram descritos no Quadro 1 com elementos que compõem os estudos analisados. 
Quadro 2: Características dos artigos analisados no período de 2016 a 2021.

\begin{tabular}{|c|c|c|c|c|c|}
\hline Título & Autoria & Ano & Objetivo & Métodos & Considerações Finais \\
\hline $\begin{array}{l}\text { Percepções } \\
\text { enfermeiros de } \\
\text { atenção primária à } \\
\text { saúde sobre o } \\
\text { cuidado a paciente } \\
\text { oncológicos }\end{array}$ & $\begin{array}{l}\text { Anne Fayma Lopes } \\
\text { Chaves; } \\
\text { Uquiana Lucas Pereira; } \\
\text { Alexsandro Monteiro da } \\
\text { Silva; } \\
\text { Luana Nunes Caldini; } \\
\text { Luana Cavalcante Lima; } \\
\text { Hérica Cristina Alves de } \\
\text { Vasconcelos }\end{array}$ & 2020 & $\begin{array}{l}\text { Conhecer as percepções de } \\
\text { enfermeiros sobre o cuidado a } \\
\text { pacientes oncológicos na ABS }\end{array}$ & $\begin{array}{l}\text { Pesquisa } \\
\text { descritiva } \\
\text { com } \\
\text { abordagem } \\
\text { qualitativa }\end{array}$ & $\begin{array}{l}\text { Os enfermeiros participantes } \\
\text { visualizaram o câncer como } \\
\text { doença estigmatizante, que } \\
\text { interfere na qualidade de vida } \\
\text { de pacientes e familiares, sendo } \\
\text { importante a assistência } \\
\text { oncológica no âmbito da } \\
\text { atenção } \\
\text { principalmente, com auxílio de } \\
\text { equipe multiprofissional }\end{array}$ \\
\hline $\begin{array}{l}\text { Situações } \\
\text { vivenciadas pela } \\
\text { equipe } \quad \text { de } \\
\text { enfermagem de } \\
\text { uma Unidade } \\
\text { Oncológica }\end{array}$ & $\begin{array}{l}\text { Géssica } \\
\text { Rodrigues Monção; } \\
\text { Kesley de Oliveira } \\
\text { Reticena; } \\
\text { Maria Fernanda } \\
\text { Gomes; } \\
\text { Estela Maris } \\
\text { Cardoso; } \\
\text { Lislaine } \\
\text { Fracolli }\end{array}$ & 2019 & $\begin{array}{l}\text { Compreender as situações } \\
\text { vivenciadas por profissionais } \\
\text { de enfermagem que cuidam de } \\
\text { pacientes com câncer }\end{array}$ & $\begin{array}{c}\text { Pesquisa } \\
\text { qualitativa }\end{array}$ & $\begin{array}{l}\text { A motivação por parte das } \\
\text { profissionais de enfermagem } \\
\text { para a prestação de cuidados } \\
\text { dignos aos pacientes com } \\
\text { câncer supera as barreiras } \\
\text { existentes }\end{array}$ \\
\hline $\begin{array}{l}\text { Teorias científicas } \\
\text { de saúde no } \\
\text { cuidado ao paciente } \\
\text { oncológico: revisão } \\
\text { integrativa }\end{array}$ & $\begin{array}{l}\text { Thalita dos Santos Costa; } \\
\text { Isaura Letícia Tavares } \\
\text { Palmeira Rolim; } \\
\text { Luciana Léda Carvalho } \\
\text { Lisboa; } \\
\text { Lívia Maia Pascoal; } \\
\text { Adriana Gomes Nogueira } \\
\text { Ferreira; } \\
\text { Janielle Ferreira de Brito } \\
\text { Lima }\end{array}$ & 2020 & $\begin{array}{l}\text { Analisar o uso das teorias de } \\
\text { saúde no cuidado a pacientes } \\
\text { oncológicos, destacando os } \\
\text { principais eixos na assistência } \\
\text { de enfermagem oncológica }\end{array}$ & $\begin{array}{c}\text { Revisão } \\
\text { integrativa }\end{array}$ & $\begin{array}{l}\text { É necessário o avanço da } \\
\text { ciência no campo da } \\
\text { enfermagem oncológica e suas } \\
\text { interações com as teorias } \\
\text { científicas. }\end{array}$ \\
\hline $\begin{array}{l}\text { Transição para os } \\
\text { cuidados paliativos: } \\
\text { ações facilitadoras } \\
\text { para uma } \\
\text { comunicação } \\
\text { centrada no cliente } \\
\text { oncológico }\end{array}$ & $\begin{array}{l}\text { Jeniffer Lopes Rodrigues } \\
\text { da Silva; } \\
\text { Isabella Ribeiro Cardozo; } \\
\text { Sônia Regina de Souza; } \\
\text { Laísa Figueiredo Ferreira } \\
\text { Lós de Alcântara; } \\
\text { Carlos Magno Carvalho } \\
\text { da Silva; } \\
\text { Fátima Helena do } \\
\text { Espírito }\end{array}$ & 2020 & $\begin{array}{l}\text { Analisar como o cliente } \\
\text { oncológico avalia a } \\
\text { comunicação na transição para } \\
\text { os cuidados paliativos; } \\
\text { Identificar suas necessidades e } \\
\text { preferências acerca dessa } \\
\text { comunicação relacionadas ao } \\
\text { seu prognóstico, tomada de } \\
\text { decisão e participação familiar; } \\
\text { apresentar proposta de ações } \\
\text { facilitadoras para comunicação } \\
\text { na transição para os cuidados } \\
\text { paliativos, a partir das } \\
\text { necessidades e preferências } \\
\text { mencionadas pelo cliente }\end{array}$ & $\begin{array}{c}\text { Pesquisa } \\
\text { convergente } \\
\text { assistencial }\end{array}$ & $\begin{array}{l}\text { Será a partir da priorização das } \\
\text { ações de escuta ativa, do } \\
\text { acolhimento, respeito à } \\
\text { autonomia e utilização de } \\
\text { linguagem clara e acessível que } \\
\text { o profissional criará vínculo } \\
\text { necessário e obterá mais êxito } \\
\text { com realizar uma comunicação } \\
\text { nas necessidades e preferências } \\
\text { do cliente oncológico }\end{array}$ \\
\hline $\begin{array}{l}\text { Atuação do } \\
\text { enfermeiro da } \\
\text { atenção primária à } \\
\text { saúde na assistência } \\
\text { oncológica: revisão } \\
\text { integrativa }\end{array}$ & $\begin{array}{l}\text { Geize Rocha Macedo de } \\
\text { Souza; } \\
\text { Luiza Helena de Oliveira } \\
\text { Cazola; } \\
\text { Ranata Palópoli Picoli }\end{array}$ & 2018 & \begin{tabular}{lcr} 
Identificar & a & \multicolumn{2}{c}{ atuação } & do \\
enfermeiro & da & Atenção \\
Primária à & Saúde & na atenção \\
oncológica, & a & partir \\
evidências & na & literatura \\
científica & &
\end{tabular} & $\begin{array}{c}\text { Revisão } \\
\text { integrativa }\end{array}$ & $\begin{array}{l}\text { Para a assistência de } \\
\text { enfermagem oncológica é } \\
\text { substancial aliar o } \\
\text { conhecimento com a prática } \\
\text { clínica, entrada no holismo e } \\
\text { no acompanhamento } \\
\text { individualizado de maneira a } \\
\text { permitir o seu envolvimento } \\
\text { em atividades assistenciais, de } \\
\text { educação em saúde e de ações } \\
\text { de prevenção e controle na } \\
\text { Atenção Primária à Saúde }\end{array}$ \\
\hline
\end{tabular}

Fonte: Autores.

Após análise dos artigos selecionados neste estudo foi construída a seguinte categoria. 
Categoria 1: A dificuldade encontrada por profissionais enfermeiros no cuidado ao paciente oncológico.

Tradicionalmente os primeiros passos elaborados para o refreamento do câncer ainda são muito novos quando igualados às demais doenças, tendo sido aconselhados, quase unicamente, para o a parte diagnóstica e terapêutica da patologia. Desta maneira, as práticas voltadas para a prevenção e orientação dos cidadãos acabam sendo afetadas. $\mathrm{O}$ que possibilita entender como o câncer transformou-se uma doença receada por todos, vinculadas a lendas e opiniões que nasceram da escassez de informações e entendimento por parcela da sociedade. (Rolim et al., 2020).

Vista como instrumento essencial para promover uma assistência individualizada e eficaz, o diálogo é uma das colunas de extrema relevância dos métodos de paliação. Refere-se a uma prática indispensável frente à degradação da patologia, especialmente na passagem para a assistência paliativa, pois concede ao paciente dividir seus receios, aflições e incertezas, facilitando na solução de embates, oferecendo o domínio pertinente de manifestações sintomáticas, além de favorecer a percepção das verdadeiras demandas do paciente. (Silva et al., 2020).

Frente à realidade do dia a dia da equipe diante dos clientes oncológicos, a condição da dor é de custosa aprovação, porque inclui requer se colocar no lugar do outro e sensibilidade, gerando pontos de descontentamento e frustração por parte desses profissionais. (Monção et al., 2019).

A enfermagem tem em como fundamento o dever com a integralidade, o qual se realiza através do amparo, conexão e comunicação e se segura no reconhecimento das insuficiências na assistência exposta pelos usuários e sua família, por ser uma ação centralizada no indivíduo e não exclusivamente na metodologia. (Souza, Cazola e Pícoli., 2018).

Nessas circunstâncias que aparece à assistência paliativa, os quais são apresentados aos indivíduos que experienciam uma patologia grave que prejudique a vida e, baseado no reconhecimento, análise e terapêutica da dor e outras manifestações físicas, psicológicas, sociais e religiosas, com finalidade de proporcionar melhor condição de vida ao cliente, a partir essencialmente de um bom diálogo, através do qual nos possibilita identificar e entender as suas demandas. (Silva et al., 2020).

Nessa fase de transição dos cuidados curativos para os paliativos, comunicar o avanço da doença e a impossibilidade de cura ainda tem sido uma das tarefas mais difíceis, se não a mais penosa para o profissional de saúde, devido à possibilidade de suscitar sentimentos diversos ao cliente, como medo do sofrimento, da dor física e da solidão, contribuindo para um sofrimento biopsicossocial. (Silva et al., 2020).

Notou-se que uma das maiores barreiras encontradas pelos profissionais enfermeiros diante do cuidado aos pacientes com câncer, são muitas vezes as restrições dos exames dispostos e das solicitações disponíveis para o cuidado integral; além da exigência de didática permanente sobre o tema. (Chaves et al., 2020).

Trabalhadores da saúde também presenciam mortes frequentemente sem privilégio de poder expressar seus sentimentos, precisando procurar maneiras para enfrentar essas dores. Logo, a criação e a aprovação de cada trabalhador podem acarretar a elevação de adoecimento e a ocorrência de depressão, por motivo de sentir-se impotente e frustrado. Isso vem colaborando para o crescimento da síndrome de Burnout, cansaço emocional, perda da personalidade e redução do sucesso particular entre profissionais. (Monção et al., 2019).

O bem-estar é primordial para o homem, mas para a maior parte dos indivíduos com câncer esse bem estar (ou essa vertente) vai aos poucos reduzindo, sendo fundamental que o enfermo receba um cuidado satisfatório quanto à assistência, tanto dos familiares como da equipe que o assiste, objetivando estrutura física e psicológica e para que possa completar cada etapa do processo. (Chaves et al., 2020).

O cuidado aos clientes com câncer exige competências técnico-científicas dos profissionais de enfermagem de maneira a conduzir maior eficácia na assistência ao tratamento, devendo compreender a patologia, seus pareceres, encargos de apoio e fins, pois estes se deparam até mesmo em casa. (Souza, Cazola e Pícoli., 2018). 
A pesquisa indica que, ao experimentar cotidianamente a aflição e declinação dos clientes, a equipe vivencia um peso afetivo, obstando a segmentação entre particular e profissional, causando desapontamento, a todos compreendidos no contexto. Porém, de acordo com as vivências obtidas, os trabalhadores entendem que o óbito é algo natural e orgânico do homem. (Monção et al., 2019).

\section{Considerações Finais}

A área oncológica é uma especialização constituída por diversos obstáculos desde a investigação, cirurgias, modelos terapêuticos e as contínuas inovações médicas, o que exige uma preparação dos profissionais de saúde, pois é de grande complexidade científica e técnica.

É válido enfatizar que a aparição do profissional enfermeiro nos ambientes que admite pacientes com câncer é de extrema importância, sendo essencial sua qualificação, pois a base prática e tecnológica, controle, conduta, compromisso e atuação clínica contribui para a organização do atendimento aos pacientes e na administração e gerência da equipe.

A partir desta pesquisa, notou-se que os motivos que dificultam a assistência do profissional enfermeiro abrangem as tarefas burocráticas, os problemas em enfrentar o processo terminal do cliente oncológico e o vínculo com a família. Além da ausência de reconhecimento por parte das empresas e gestão a escassez de instrução permanente e a carência de planejamento organizacional para reduzir os efeitos físicos e emocionai para os trabalhadores, usuários e familiares.

Determinadas táticas para diminuir essas condições estão conectadas com a quantidade reduzida de trabalhadores, infraestrutura material e aparelhos e outros. Bem como, retorno positivo profissional por parte da instituição como planejamento de carreira para um aprimoramento profissional dos trabalhadores, ambiente organizacional para exposição dos receios e desejos, orientação em saúde continuada e permanente com o objetivo de capacitar profissionalmente e estimular a enfrentar o processo de luto, incluindo assistência psicológica.

Pesquisas iminentes precisam ser realizadas com a finalidade de sofisticar o convívio do profissional enfermeiro em sua unidade de trabalho, que assiste usuários com câncer para reestabelecer o bem-estar de todos os trabalhadores que atendem o cliente oncológico e seus familiares, o que por consequência intervém no padrão e na humanização do cuidado ofertado.

\section{Referências}

Bardin, L. (2016). Análise de conteúdo. Editora Almedina.

Chaves, A. F. L. et al. (2020) percepções de enfermeiros da atenção primária à saúde sobre o cuidado a pacientes oncológicos. Enferm. Foco 11 (2): 91 -97 91. Ceará, Brasil.

Cyriaco, A. F., Nunn, D., Amorim, R. F., Falcão, D. P., \& Moreno, H. (2017). Pesquisa Qualitativa: conceitos-chave e um breve panorama de sua aplicação em geriatria/gerontologia. Envelhecimento, 11(1), 4-9.

Gerhardt, T. E., \& Silveira, D.T. (2009). Métodos de pesquisa. UFRGS.

Goldenberg, M. (1997). A arte de pesquisar. Record.

Mendes, K., Silveira, R. C., \& Galvão, C. M. (2008). Revisão integrativa: método de pesquisa para a incorporação de evidências na saúde e na enfermagem. Texto Contexto Enfermagem, Florianópolis, 17(4): 758-64.

Minayo, M. C. (2011). Pesquisa social. Teoria, método e criatividade. Editora Vozes.

Monção, G. F. R. et al (2019). Situações Vivenciadas pela Equipe de Enfermagem de uma Unidade Oncológica. Revista Brasileira de Cancerologia, 65(1): e11325 .

Polit, D.F., \& Beck, C.T. (2011). Delineamento de Pesquisa em Enfermagem. Artmed.

Rolim, I. L. T. P. et al (2020). Teorias científicas de saúde no cuidado ao paciente oncológico: revisão integrativa. Revista de Enfermagem do Centro-Oeste Mineiro, 10/3654.

Santos, C. M., Pimenta, C. B., \& Nobre, M. R. (2007). The PICO Strategy for there search question construction and evidence search. Ver. Latino-Am. Enfermagem, 15(3), 508-511. 
Research, Society and Development, v. 10, n. 15, e172101522784, 2021

(CC BY 4.0) | ISSN 2525-3409 | DOI: http://dx.doi.org/10.33448/rsd-v10i15.22784

Silva, J. L. R. da, et al (2020). Transição para os cuidados paliativos: ações facilitadoras para uma comunicação centrada no cliente oncológico. Reme: Revista Mineira de Enfermagem, 24, e1333.

Silva, V. C. E., \& Zago, M. M. F. (2005). A revelação do diagnóstico de câncer para profissionais e pacientes. Revista Brasileira de Enfermagem, 58(4), 476480 .

Souza, G. R. M. de, et al (2018). Atuação do enfermeiro da atenção primária à saúde na assistência oncológica: revisão integrativa. Cogitare Enfermagem, 23(4), e58152.

Souza, M. T., Silva, M. D., \& Carvalho, R. (2010). Revisão integrativa: o que é e como fazer. Einstein, 8(1), 102-106.

Stumm, E. M. F., Leite, M. T., \& Maschio, G. (2008). Vivências de uma equipe de enfermagem no cuidado a pacientes com câncer. Cogitare Enfermagem, $13(1), 15-82$. 